\title{
Basic science research in medicine
}

\author{
Pandey AS \\ Department of Biochemistry, Kathmandu Medical College, Duwakot
}

The utility of research in basic sciences is not discernible to the casual observer, as most of it is undertaken to answer scientific questions rather than with the goal to cater to the needs of a particular discipline. The relevance of basic sciences to medicine is not immediate, as decades pass before the relevance of a particular research can be recognized. One recent example is Robert Edwards of the University of Cambridge, who won the Nobel Prize in Physiology andMedicine for pioneering in vitro fertilization. His work began in the 1950's and throughout his research, "By a brilliant combination of basic and applied medical research, Edwards overcame one technical hurdle after another in his persistence to discover a method that would alleviate infertility", stated the Karolinska Institute. Earlier in 2009, the Nobel Prize in Physiology or Medicine was shared amongst Basic Scientists for work on telomeres that began in the 1970's just for reasons of curiosity from the side of the researchers. In trying to answer basic scientific questions, they discovered the enzyme telomerase and its role in determining the telomere size, which later come to be associated with aging, cardiovascular diseases, diabetes, and cancer.

'Basic science' may be any fundamental science that describes the most basic objects and forces; the relation between them; and the laws governing them; in such a way that all other phenomenon may be in principle derived from them following the logic of scientific reductionism. These include physics; chemistry; biology; mathematics; geology; and the list continues. Basic medical sciences in medicine constitutes anatomy, physiology, biochemistry, pharmacology, pathology and microbiology. These basic sciences are incorporated into medical education to provide an understanding of the scientific basic of disease, its diagnosis, and treatment. A knowledge and understanding of the scientific principles that govern human biology provides medical doctors not only with a rationale for the contemporary practice of medicine, but also with a framework to incorporate new knowledge into their practices in the future. ${ }^{1}$ There is a gap in the understanding of how basic science knowledge actually influences medical practice. As a result, there is little or no appreciation of the years of work in basic sciences that have radically improved diagnosis, treatment, disease management and hospital care. This has given rise to the concept of "translational research" which is defined as "the process of applying ideas, insights and discoveries generated through basic scientific inquiry to the treatment or prevention of human disease" ${ }^{\prime 2}$. Translational research is supposed to bridge the gap between the basic science research done in the laboratory and the application of the discoveries hence made to clinical practice and the community. Nevertheless, without basic science research, translational research will gradually ebb and fade.

Examples of contributions of basic science research to medicine are abound; antiretroviral therapy for AIDS has been possible because of an understanding of the underlying mechanisms of replication of retroviruses. The antiretroviral drugs have also undergone an evolutionary change to become more precise, stronger and more effective. This was possible by determining the crystal structures of these drugs bound to their targets, working out the potential bonds that would improve binding, and redesigning drugs that were more combative towards the virus.

Basic science research seeks to go deep into a problem which may or may not be medically relevant. It aims to define at the molecular level the cause of the problem and hence forms the basis of finding a solution to the problem. In humans, the physiological and biochemical mechanisms that run the body, are deciphered in the lab through studies on cells and their constituents. 
Similarly, bacterial studies utilize the identification of relatives of infectious bacteria that themselves are not virulent, to study the mechanisms of the disease causing bacteria. This ensures the safety of the personnel working with these strains while providing important detail about the cellular mechanisms of infectious bacteria. Human cell lines that can propagate are being used to study the complicated mechanisms of differentiation, drug metabolism, cancer and mutations. Better and more specific drugs are being created by studying the crystal structures of protein targets, as mentioned above in the case of drugs for HIV. It needs to be appreciated that not all research as these arise to address a disease. With the consequences of changes in our environment becoming obvious in terms of impact on our health and well being, all that constitutes the environment becomes relevant science. At the core of basic science research, the inquisitiveness of the scientist should remain unharmed and untouched, as this is what will determine the technological and academic advances in the future.

In Nepal, basic science research is sadly non-existent. In what is deemed the absence of a "direct benefit" to the community, or a direct utilization in a hospital, research has been reduced largely to epidemiology and health surveys. The scientists of Nepal who want to be little else other than scientists, are merely limiting their careers. The questions that arise in the minds of Nepali scientists have to be silenced, unless they themselves decide to undertake their objectives. This is a formidable task in any part of the world. As a people and culture, we cannot progress unless the curious minds amongst us are nurtured and given an opportunity to explore problems and discover solutions. With the constraints in which a basic scientist will have to work in Nepal, it is logical to assume that the outcome of their ventures will be quite different from that produced by the developed world.

Caroline Greider, who shared the Nobel Prize in Physiology or Medicine for her role in the discovery of a new enzyme "telomerase" thanked the Nobel Foundation for the award and emphasized that the recognition highlights the value of "discoveries driven by pure curiosity. We had no idea when we started this work that telomerase would be involved in cancer, but were simply curious about how chromosomes stayed intact." ${ }^{3}$ Her work utilized a single-celled, pond-dwelling organism called Tetrahymenas - rather than primates or humans - as these were the best test system because Tetrahymena contain "more like 40,000 chromosomes, compared to our 23 pairs" and thus have far more chromosome ends to study.

Short of a century ago, in 1921, Marie Curie in her famous speech at Vassar College in New York ${ }^{4}$ had said,

"........But we must not forget that when radium was discovered no one knew that it would prove useful in hospitals. The work was one of pure science. This is proof that scientific work must not be considered useful only from the perspective of its outcomes, but for itself; for the beauty of science; and the chance that a scientific discovery may become like the radium a benefit for humanity."

\section{REFERENCES}

1. Association of American Medical Colleges. Medical Schools Objectives Project Report IV, Contemporary Issues in Medicine: Basic Science and Clinical Research. Washington, US [Publisher Unknown]; 2001 Aug. p. 5.

2. Ninds Exploratory [Internet]. USA National Institute of Health; 2002 Jul 26 [cited 2011 Jan 11]. Available from: http://grants1.nih.gov/grants/guide/pa-files/PAR-02-138.html

3. John Hopkins Medicine [Internet]. [Place Unknown]: [Publisher Unknown] 2009 [updated 2009 Oct 05; cited 2011 Jan 11]. Available from: http://www.hopkinsmedicine.org/news/media/releases/telomere_expert_carol_greider_ shares_2009_nobel_prize_in_physiology_or_medicine

4. Famous Quotes [Internet]. [Place Unknown]: [Publisher Unknown] Available from: http://www.famousquotes. me.uk/speeches/Marie-Curie 